\title{
Criminologie
}

\section{Travailler pour survivre : exploration du travail des jeunes de la rue}

\author{
Jeff Karabanow, Jean Hughes et Sean Kidd
}

Volume 43, numéro 1, printemps-été 2010

Les jeunes et la rue

URI : https://id.erudit.org/iderudit/044049ar

DOI : https://doi.org/10.7202/044049ar

Aller au sommaire du numéro

\section{Éditeur(s)}

Les Presses de l'Université de Montréal

ISSN

0316-0041 (imprimé)

1492-1367 (numérique)

Découvrir la revue

Citer cet article

Karabanow, J., Hughes, J. \& Kidd, S. (2010). Travailler pour survivre : exploration du travail des jeunes de la rue. Criminologie, 43(1), 7-29. https://doi.org/10.7202/044049ar
Résumé de l'article

Le présent article explore les diverses formes que prennent l'emploi et les " petits boulots " dans la vie des jeunes de la rue. L'étude a été faite à Halifax, au Canada, et porte sur un matériel tiré d'entretiens menés auprès de 34 jeunes. Les résultats de l'enquête suggèrent que l'économie du travail à laquelle doivent faire face les jeunes de la rue revêt un aspect autant formel qu'informel. Ils montrent aussi que ces jeunes sont motivés mais vivent aussi des enjeux tant personnels qu'environnementaux. La plupart des jeunes interrogés réalisent des activités informelles rémunératrices parmi lesquelles figurent entre autres la mendicité, la pratique du squeegee (du nom anglais de la raclette servant à nettoyer les pare-brise), et l'art ambulant, la vente de créations artistiques, la composition et la déclamation de poèmes, la diffusion de blagues et autres activités créatrices. La diversité de ces activités témoigne de l'esprit d'entreprise et de la capacité de résilience de cette jeunesse itinérante. Pour survivre, les jeunes sans-abri sont en effet prêts à entreprendre un grand nombre de tâches souvent peu valorisées dans notre société. À vrai dire, il leur est surtout difficile d'accéder à un travail socialement reconnu. Pourtant, la société, en s'inscrivant dans une logique de criminalisation de certains types de pauvreté, réprime le plus souvent les activités informelles qu'adoptent les jeunes comme stratégies de survie.
Ce document est protégé par la loi sur le droit d'auteur. L’utilisation des services d'Érudit (y compris la reproduction) est assujettie à sa politique d'utilisation que vous pouvez consulter en ligne.

https://apropos.erudit.org/fr/usagers/politique-dutilisation/ 


\title{
Travailler pour survivre : exploration du travail des jeunes de la rue
}

\author{
Jeff Karabanow \\ School of Social Work, Dalhousie University \\ jeff.karabanow@dal.ca \\ Jean Hughes \\ School of Nursing, Dalhousie University \\ jean.hughes@dal.ca \\ Sean Kidd \\ Center for Addictions and Mental Health \\ sean_kidd@camh.net
}

\begin{abstract}
RÉSUMÉ - Le présent article explore les diverses formes que prennent l'emploi et les "petits boulots» dans la vie des jeunes de la rue. L'étude a été faite à Halifax, au Canada, et porte sur un matériel tiré d'entretiens menés auprès de 34 jeunes. Les résultats de l'enquête suggèrent que l'économie du travail à laquelle doivent faire face les jeunes de la rue revêt un aspect autant formel qu'informel. Ils montrent aussi que ces jeunes sont motivés mais vivent aussi des enjeux tant personnels qu'environnementaux. La plupart des jeunes interrogés réalisent des activités informelles rémunératrices parmi lesquelles figurent entre autres la mendicité, la pratique du squeegee (du nom anglais de la raclette servant à nettoyer les pare-brise), et l'art ambulant, la vente de créations artistiques, la composition et la déclamation de poèmes, la diffusion de blagues et autres activités créatrices. La diversité de ces activités témoigne de l'esprit d'entreprise et de la capacité de résilience de cette jeunesse itinérante. Pour survivre, les jeunes sans-abri sont en effet prêts à entreprendre un grand nombre de tâches souvent peu valorisées dans notre société. À vrai dire, il leur est surtout difficile d'accéder à un travail socialement reconnu. Pourtant, la société, en s'inscrivant dans une logique de criminalisation de certains types de pauvreté, réprime le plus souvent les activités informelles qu'adoptent les jeunes comme stratégies de survie.
\end{abstract}

MotS-CLÉs - Jeunes de la rue, emploi, stratégie de survie, déviance, marginalité, économie informelle.

Traduit de l'anglais par Rabia Mzouji.

Criminologie, vol. 43, $\mathrm{n}^{\circ} 1$ (2010) 


\section{Introduction}

\section{Les économies informelles}

Les économies formelles et informelles existent partout dans le monde. Les premières comprennent le travail légal et légitime, celui qui est recensé et à ce titre imposé. Les économies informelles, elles, tendent à s'en différencier, tant par la manière dont le travail s'y organise que par la nature des biens et services qui y sont échangés (Losby et al., 2002; Schneider, 2002). La prestation d'un service payé comptant (pour avoir gardé des enfants, pour avoir fait des heures supplémentaires au travail, etc.) et non comptabilisé dans la déclaration d'impôt serait par exemple considérée comme du travail informel. Comme les rémunérations touchées n'apparaissent pas dans la déclaration, de tels exemples sont perçus comme illégitimes du point de vue de la régulation formelle du travail, mais on reste dans l'ordre du légal. On pourrait a priori tenir le même raisonnement pour la mendicité puisqu'elle aussi est légale au Canada. Il y a pourtant une différence de taille puisqu'elle est considérée illégale si les gains ne sont pas rapportés. La part illégale des économies informelles inclut des activités criminelles comme le trafic de drogue, le travail du sexe, la vente de biens volés et le vol (Gaetz et O'Grady, 2002; Losby et al., 2002; Schneider, 2002).

Alors que les économies informelles étaient presque exclusivement associées aux pays en voie de développement, leur présence est maintenant de plus en plus reconnue dans le contexte des nations industrialisées (Reimer, 2000; Schneider, 2002; Vogel, 2006). À ce titre, la mondialisation, notamment dans le cadre de la mise en œuvre de l'Accord de libre-échange nord-américain (ALENA), joue un rôle très important dans l'émergence des économies informelles au Canada et aux ÉtatsUnis. Si l'ALENA a semblé profiter à l'économie mexicaine en assurant une exportation de bas salaires et en rendant ses biens compétitifs sur le marché, il a grandement endommagé le secteur de la manufacture, à petite comme à grande échelle, et ce tant au Canada qu'aux États-Unis. À la suite de l'implantation de l'ALENA, les industries manufacturières canadiennes et étatsuniennes se sont bientôt retrouvées incapables de concurrencer les biens importés à bas salaire du Mexique et ont été contraintes à de nombreuses suppressions d'emploi et de fermetures d'usines. Or, les industries manufacturières et à bas salaire sont souvent la seule option possible pour gagner sa vie quand on a un profil de travailleur sous-qualifié. Au total, les États-Unis auraient perdu 
3,4 millions d'emplois manufacturiers depuis 2001. Dans le même temps, aucune nouvelle économie n'a émergé pour remplacer les emplois perdus (Scott, 2008). Quant au Canada, il a connu un sort identique (Faux et al., 2006).

Les économies informelles apparaissent quand les temps sont durs. Les individus, les communautés ou les populations qui ont besoin d'argent réfléchissent à des manières d'acquérir ce dont ils ont besoin. Quand on pense aux économies informelles, on pense souvent aux économies du marché noir, clandestines, souterraines, invisibles; bref, à toutes ces économies qui deviennent synonymes de stratégies de survie pour les nombreux travailleurs qui ne peuvent pas trouver d'emploi dans l'économie formelle (Losby et al., 2002; Vogel, 2006). Loin de contrecarrer ces réalités économiques difficiles, le gouvernement canadien a au contraire réduit son rôle de fournisseur de services sociaux pourtant destinés à compenser les tendances à la baisse de la conjoncture économique (Faux et al., 2006). Le gouvernement fédéral a en outre opéré des changements majeurs dans sa façon d'octroyer et de construire des habitations abordables pour les gens disposant de faibles revenus. Une politique qui a contraint des populations marginalisées ayant peu d'autres choix à vivre dans la rue et à s'engager dans l'économie informelle (Gaetz et O'Grady, 2002; Hunter et al., 2009). Parmi les participants typiques à ce genre d'économie, on retrouve les immigrants illégaux, les travailleurs qui ne peuvent pas être officiellement déclarés pour toutes sortes de raisons (par exemple, le fait d'avoir un casier judiciaire) ou encore les travailleurs qui sont sans emploi en raison d'un chômage structurel et systémique.

L'économie informelle et les expériences de vie des jeunes de la rue

Les jeunes de la rue sont fréquemment impliqués dans les économies informelles. Les obstacles qu'ils rencontrent pour bénéficier d'un emploi formel sont en effet nombreux: l'instabilité résidentielle, l'âge, le manque d'éducation et souvent l'absence de documents administratifs en ordre (Gaetz et O'Grady, 2002; Karabanow, 2004b). Les jeunes de la rue sont définis comme de jeunes gens (ils ont généralement entre 16 et 24 ans) qui n'ont pas leur propre logement et qui, à la place, passent un temps significatif et mettent une énergie considérable dans la «rue» (les ruelles, les parcs, les devantures de magasins, les bennes, etc.), dans 
les squats (habituellement situés dans les immeubles abandonnés), dans les abris et les centres pour jeunes et/ou avec des amis (ils dorment les uns chez les autres) (Dachner et Tarasuk, 2002; Karabanow, 2004a; Halifax Regional Municipality, 2005). La question de la jeunesse itinérante canadienne est souvent intrinsèquement liée à celle des violences familiales, de la pauvreté, de l'instabilité et du traumatisme, des maladies mentales apparues durant l'enfance ou à l'adolescence (Coates, 2000; Novac et al., 2002; Karabanow, 2004a, 2006, 2008; Hughes et al., 2005). Les histoires d'expériences problématiques et déshumanisantes sont monnaie courante dans les centres de réadaptation et de protection de la jeunesse et une grande majorité de ces jeunes arrive dans la rue par désespoir ou en tout cas avec la conviction que leur vie y sera plus sûre et plus acceptable que la vie qu'ils fuient (Alleva, 1988; Auerswald et Eyre, 2002; Karabanow, 2004a). Une fois dans la rue, nombre de ces jeunes recourent à des activités risquées et souvent illégales pour subvenir à leurs besoins (Auerswald et Eyre, 2002; Karabanow, 2004a). De telles pratiques les mettent un peu plus encore au ban de la société et rendent plus difficile encore leur intégration, déjà compromise par des problématiques telles que les addictions et les maladies, physiques et mentales, mais aussi la présence répétée de l'intervention pénale dans leur vie (Novac et al., 2002; Durham, 2003; Hughes et al., 2005 ; Karabanow et al., 2005 ; Karabanow, 2006, 2008 ; Karabanow et al., 2007).

L'engagement des jeunes de la rue dans l'économie informelle est trop souvent discuté dans son seul rapport avec les économies des activités criminelles (trafic de drogue, commerce du sexe, vols) (Hagan et McCarthy, 1997; Baron, 2004). Ce faisant, la perception commune et stigmatisante que l'activité criminelle est le seul ou en tout cas le premier moyen de subsistance pour la plupart des jeunes sans-abri, sinon pour tous, se renforce. Comprises sous la forme d'activités illégales pour taxes non payées, les économies informelles jouent en fait un rôle très important dans les stratégies de survie quotidiennes de la plupart des jeunes de la rue (Gaetz et O'Grady, 2002). Il est important de noter que bon nombre de recherches criminologiques nord-américaines qui portent sur les manières informelles auxquelles recourent les jeunes de la rue pour gagner de l'argent traduisent presque toujours cette question en termes de crime plutôt qu'en termes de travail (entendu comme moyen d'obtenir de l'argent), et ce, alors que gagner sa vie dans un secteur formel (où, pour rappel, le revenu est rapporté et taxé) est 
considéré comme un travail ou un emploi (Gaetz et O'Grady, 2002). L'essentiel de la recherche vise dès lors à comprendre comment les jeunes de la rue deviennent «criminellement impliqués» plutôt que d'appréhender les revendications d'autonomie qu'ils formulent (indépendamment d'une quelconque nécessité de rentrer dans un processus criminel). En outre, la traduction en «crimes» d'activités de la rue comme la pratique du squeegee ou la mendicité ne permet pas de comprendre la motivation et l'enjeu de survie qui accompagnent la poursuite de telles activités entrepreneuriales. Hagan et McCarthy (1997) suggèrent qu'une majorité des jeunes de la rue préfère ne pas s'engager dans certaines stratégies illégales (prostitution, trafic de drogues) pour obtenir de l'argent. De tels résultats laissent donc entendre que ces jeunes, communément vus comme «déviants» ou «délinquants» souhaitent souvent un emploi en règle, contrairement à ce que pense une grande partie de l'opinion publique (Hagan et McCarthy, 1997; Baron, 2001 ; Gaetz et O'Grady, 2002; Karabanow, 2006, 2008).

\section{La présente recherche}

Dans notre étude, nous avons examiné les récits de jeunes sans-abri qui s'engagent dans les économies formelles et informelles. L'examen exploratoire et qualitatif des expériences de ces jeunes a permis de relever des points d'articulation entre leurs identités professionnelles, le sens que les jeunes donnent aux diverses formes de travail et leurs réalités socioéconomiques (voir Karabanow et al., 2009, pour le rapport complet de l'étude). Une approche qualitative a été entreprise avec deux considérations à l'esprit. La première, c'est que seul un petit nombre d'études avaient été conduites sur la problématique de l'emploi pour les jeunes de la rue (par exemple, Baron et Hartnagel, 2002; O'Grady et Gaetz, 2004), aucune étude n'avait analysé en profondeur et surtout en priorité les expériences vécues à la lumière du débat entre économies formelles et informelles. La seconde, c'est que les questions examinées dans cette étude sont complexes avec des interactions entre 1) contextes, structures et normes communautaires à replacer dans le cadre des économies formelles et informelles, 2) expériences individuelles des jeunes, et 3) significations à retraduire dans les sous-cultures des jeunes. Compte tenu de cette situation, une approche qualitative exploratoire montre non seulement la nécessité de parfaire nos connaissances dans un champ encore peu étudié mais aussi l'intérêt de mieux comprendre la manière 
complexe dont les structures liées à l'emploi s'articulent à divers systèmes de sens.

\section{Démarche méthodologique}

Après approbation du comité d'éthique de l'Université de Dalhousie, nous avons mené des entretiens qualitatifs approfondis et semi-structurés avec 34 jeunes à Halifax. Des entrevues ont été réalisées auprès de jeunes de 16 à 24 ans qui ont été identifiés comme jeunes de la rue ou jeunes sans-abri. La moyenne d'âge des participants était de 21 ans: $39 \%$ de l'échantillon était constitué de femmes, $16 \%$ se déclaraient comme homosexuels, lesbiennes ou bisexuels, $44 \%$ définissaient leur ethnicité comme caucasienne et $18 \%$ comme aborigène, $50 \%$ dormaient dans des conditions très précaires au moment de l'entretien. Enfin, une vaste majorité d'entre eux avait la pratique du squeegee et la mendicité comme principales sources de revenus.

Les guides d'entretien de l'équipe de recherche ont été orientés en vue de mettre en forme les récits collectés autour des aspects clés de l'emploi et des petits boulots (incluant la routine quotidienne des jeunes de la rue, leurs préférences, leurs vécus scolaires et leurs mentors, les points positifs qu'ils retiennent de leur vie, les défis qu'ils rencontrent, leurs «jobs de rêve», etc.). Les entretiens ont été menés dans un café ou un centre de jour, et ont duré en moyenne une heure. Ils ont été enregistrés, retranscrits et gardés anonymes. Chaque participant a été interviewé une fois et a reçu une petite somme d'argent. Recourant à la théorie ancrée (grounded theory), le devis de recherche s'appuyait sur le contexte communautaire naturel et tentait moins de vérifier des hypothèses que de les bâtir à partir des données émergentes. C'est donc la lecture qu'il faut faire des arguments que nous avancerons ici. L'analyse de la théorie ancrée comporte un processus en vue d'identifier dans les entretiens tant des thèmes semblables que des thèmes différents, et ce, tout en présentant des récits dont la conceptualisation est tirée de données lues à travers une codification ouverte, axiale et sélective (Strauss et Corbin, 1990). Ce processus facilite une lecture des données en catégories et thèmes conceptuels spécifiques puisqu'il réorganise les données de façon à lier catégories primaires et thèmes auxiliaires dans le cadre d'une analyse causale. Enfin, ce processus construit un récit théorique façonné par l'intégration des données et la construction des catégories. 
Cette étude a été élaborée et mise en œuvre en collaboration avec un centre de jour présenté comme une alternative communautaire et appelé $A R K$ Outreach, à Halifax, en Nouvelle-Écosse. Pour nous assurer que le projet soit véritablement ancré dans la communauté et les expériences vécues des jeunes gens, nous avons invité trois jeunes de la rue qui fréquentaient ARK à faire partie du projet à titre de comité consultatif. Ces trois jeunes nous ont accompagnés dans le recrutement des participants, dans l'analyse des données, dans les réévaluations successives des résultats et enfin, dans les présentations scientifiques et communautaires de notre travail.

Un échantillonnage de convenance a été employé. Les jeunes invités à participer à l'étude présentaient les critères suivants: avoir entre 16 et 24 ans (soit la tranche d'âge la plus commune quand on parle des jeunes de la rue), être identifiés comme sans-abri et/ou comme vivant dans la rue. Durant l'été (plus précisément les mois de juillet/août), les jeunes qui «voyageaient ${ }^{1} »$ ou qui se baladaient aux alentours ont été recrutés pour le premier tour de la collecte de données. À l'automne, ce sont les jeunes qui étaient davantage «établis» et qui bénéficiaient probablement d'un travail formel qui ont été recrutés et interrogés. Cette stratégie a été entreprise de façon à diversifier notre échantillon.

\section{Résultats}

Les participants à l'étude ont clairement avancé la raison pour laquelle ils s'engageaient dans des activités à la fois formelles et informelles: c'était pour eux la seule manière de survivre à la précarité inhérente à la vie de la rue. Si la majorité des jeunes gens interrogés avaient des expériences de travail tant formelles qu'informelles, c'était le deuxième type d'activité qui restait de loin le plus fréquent: pratique du squeegee, mendicité, etc. Trois récits principaux peuvent être tirés des données: 1) la vie de rue comme travail ; 2) des enchevêtrements significatifs entre activités formelles et informelles; et 3) le travail comme construction identitaire pour les jeunes qui vivent dans la pauvreté.

1. Les «voyageurs» constituent une sous-population des jeunes de la rue, sous-population dont les membres se caractérisent généralement par leurs déplacements d'Est en Ouest au Canada, ne restant jamais plus que quelques mois dans chacune des villes où ils s'arrêtent. "Voyager» est une activité estivale pour la plupart d'entre eux. ARK a estimé important que l'étude s'intéresse aux expériences d'une telle sous-population. 


\section{La rue comme travail}

La routine des jeunes vivant sans domicile et engagés dans un travail informel n'est pas significativement différente de la routine qu'exige le maintien d'un emploi formel; la différence concerne finalement surtout celui qui est chargé d'établir cette routine. Pour les jeunes interrogés, un des avantages ou attraits du travail informel consiste dans le fait de n'avoir personne d'autre que soi-même pour définir, superviser ou fixer les heures de travail, qu'il s'agisse d'imposer un début ou une fin, ou encore de décider des heures de pause:

Quand maintenant je pense à me trouver un emploi, je pense à «abandonner une bonne part de ma liberté» parce que ouais, comme tu sais, et même si ça peut sembler stupide de dire ça, quand tu trouves une job, il faut bien la faire. Tu as quelqu'un qui te dit quoi faire et tout devient alors différent de ce que tu as vécu en gagnant de l'argent par toi-même et en vivant comme tu l'entendais ${ }^{2}$. (Jeune homme de la rue, 19 ans)

Loin des préjugés qui caractérisent habituellement le travail de rue informel, les jeunes voient surtout ce dernier comme l'expression de leur créativité, de leur résilience et de leurs aptitudes d'entrepreneur, des qualités qui leur apparaissent nécessaires pour s'adapter à leurs propres réalités (celle du sans-abri, du voyageur, etc.). Ces jeunes trouvent aussi que le travail informel les aide à mieux s'adapter à d'autres types d'engagements (par exemple des rendez-vous avec des travailleurs sociaux, la tenue et le respect d'horaires pour les repas et les programmes, le repère de refuges), ne serait-ce que pour satisfaire en temps et heure leurs besoins essentiels de survie. Ceci dit, le travail informel fait entrer les jeunes sans-abri dans la sphère publique et par conséquent aussi dans celle de la charité publique, une situation qui les expose à la colère d'une société souvent peu compréhensive à leur égard:

J'ai rencontré des gens qui me haranguaient en me disant «va te faire foutre, stupide merde sans valeur, blablabla, trouve une putain de job, pourquoi ne vas-tu pas juste crever!» Ils essaient parfois même de me cracher dessus. J'ai été frappé et d'autres trucs du genre. Il y a des gens qui, de leur voiture, me jettent des objets à la tête. Juste comme ça, mon gars, ces gens m'insultent juste pour m'insulter. (Jeune homme de la rue, 17 ans)

2. Nous rappelons au lecteur que les propos des interviewés ont été exprimés en anglais. La traductrice a veillé à en faire une traduction qui reflète le mieux possible la manière de parler de ces jeunes (NDR). 
Les jeunes femmes en particulier vivent de telles histoires d'agression (la plupart, de nature sexuelle) quand elles travaillent informellement, et décrivent comment elles recourent à des stratégies pour se protéger de ce type de situations:

Si j'étais un homme, j'utiliserais probablement une pancarte pour le travail, mais vu que je suis une fille, si je tiens une pancarte en carton, les gens se font des idées malsaines sur ce qu'est mon travail. Je me sentirais bizarre de tenir une pancarte comme ça, surtout avec les pervers et ce genre de gars. (Jeune femme de la rue, 18 ans)

Se battre contre les préjugés négatifs qui entourent le travail de la rue est vu comme une "part de la job» et rester positif est la clé pour garder espoir face à de telles circonstances extrêmes: "Tu dois juste te dire que tu es au sommet du monde et ne surtout pas prêter attention à ce que les gens te disent dans la rue. Si tu réussis à ne pas te laisser atteindre, tu arriveras à être heureuse » (jeune femme de la rue, 21 ans). Expérimentant de près l'agression verbale et la menace perpétuelle de violence physique, ces jeunes se disent surtout frustrés par la méconnaissance des gens à propos de ce que vivent celles et ceux qui n'ont pas de domicile, en particulier par la méconnaissance dont ils font preuve par rapport aux exigences que suppose l'engagement dans des activités informelles.

\section{L'enchevêtrement du travail formel et informel}

La grande majorité des jeunes de la rue sont embrigadés dans du travail informel. Mais, très tôt dans les entretiens, il est devenu clair que les jeunes de la rue sont aussi engagés dans du travail formel sur plusieurs plans - chercher une job, quitter une job, avoir une job où retourner, ou tout simplement travailler en ce moment dans le secteur formel. Dans la plupart des cas, les jeunes travaillent dans une activité qui ne demande pas de réelles qualifications ou encore occupent des emplois du secteur des services. Les participants à l'étude qui ont un travail formel ont généralement une meilleure opinion d'eux-mêmes et attachent plus de valeur à l'argent qu'ils gagnent quand il est obtenu après une journée de labeur socialement perçue comme «honnête». Toutefois, la majorité des jeunes gens reconnaît que souvent, combiner absence de domicile et travail formel reste incompatible: 
J'ai travaillé parfois en étant sans-abri, mais c'est juste extrêmement dur. $\mathrm{Tu}$ travailles [un temps plein] puis tu dois aller chercher un refuge; alors que tu dors, tu as la police et d'autres personnes qui te réveillent et te mettent dehors. Après ça, tu marches dans la ville pour un autre douze heures et ensuite tu retournes travailler. Tu finis par ne plus y arriver... C'est très difficile de garder un travail quand tu es dans la rue... c'est presque impossible. À moins que tu puisses avoir un logement, mais tu ne peux pas avoir de logement sans job, et tu ne peux pas avoir de job sans logement. Bref, tu t'enfonces toujours plus dans le même cercle vicieux. (Jeune homme de la rue, 19 ans)

La nécessité d'un logement assuré, adéquat et à un prix raisonnable a été avancée par les participants comme la raison principale pour laquelle ils ne pouvaient pas garder un travail formel. Avoir un logement permet un espace pour se préparer au travail. Être capable de manger, de se reposer et de se laver sont des aspects essentiels pour garder son emploi et son employabilité. Toutefois, d'autres difficultés et inégalités systémiques barrent, elles aussi, la route à nombre de jeunes qui souhaiteraient bien gagner leur vie. Après avoir travaillé pour un chèque de paie, les jeunes doivent encore investir beaucoup d'énergie dans un travail informel pour survivre parce qu'ils n'ont pas ou ont peu d'argent (ou parce qu'ils doivent attendre plusieurs semaines pour toucher leur paie). En outre, ils doivent endurer les commentaires insultants d'un public qui part du principe que ces jeunes sont sans travail. Cette expérience est particulièrement frustrante, comme l'exprime une jeune femme :

Chaque fois que je commence une job, je dois mendier jusqu'à l'arrivée de mon premier chèque de paie. Bref, ouais, après le travail, c'est galère parce qu'après avoir travaillé une journée temps plein, je dois aller demander aux gens de la monnaie et eux me disent d'aller me trouver une job. Tu sais, c'est un peu exaspérant d'entendre ça... alors que tu travailles! (Jeune femme de la rue, 18 ans)

Travail formel et travail informel s'enchevêtrent ainsi continuellement. Très souvent, le travail informel n'est pas vu comme un choix; il est plutôt perçu comme nécessaire à la survie quand le travail formel est tout simplement inaccessible. Les motivations des jeunes de la rue pour travailler informellement sont les mêmes que celles qui les amènent à travailler dans l'économie formelle: gagner de l'argent pour survivre et ce, même s'ils considèrent la mendicité comme une activité «humiliante» et ne revêtant «aucune dignité». 


\section{La signification du travail}

Les participants à l'étude sont très conscients de la position qu'ils occupent dans la société. Certains se voient eux-mêmes comme vivant en dehors de la société, mais d'autres revendiquent en faire encore partie, en premier lieu parce que leur travail se situe clairement dans la sphère publique. Cette tension autour de la citoyenneté est un thème clé de ce que signifie le travail pour les jeunes qui ont participé à l'étude:

Tu te sens mal parce que tu es dans la rue, que tu es dehors et que tu fais de l'argent. Quand tu fais de l'argent, tu te sens mal d'en prendre à d'autres personnes mais tu dois revenir et le faire de nouveau le jour suivant. Alors que tu viens juste de le faire. (Jeune homme de la rue, 22 ans)

Toutefois, d'autres jeunes soulignent à quel point leur travail informel les a aidés à développer un contact positif avec le public. Les échanges et les conversations qu'ils ont eus dans la rue avec les passants leur ont permis d'apprendre bien des choses et leur ont donné du temps pour réfléchir à de nouvelles perspectives. De tels moments ont aidé ces jeunes à se bâtir un sentiment d'appartenance, même en tant que marginaux.

Bien que le travail formel et le travail informel donnent lieu à des expériences très différentes, beaucoup de jeunes estiment que travailler dans un cadre informel n'est pas si différent que de le faire dans un contexte formel, du moins en termes d'efforts, de dévouement ou encore de motivation. Pour donner du crédit au travail informel, il faut montrer qu'il nécessite, de la part du travailleur, courage et abnégation: "[Je travaille] autant que je le ferais pour un travail normal. Je suis ici tous les jours et j'essaie vraiment de me tenir à la cohérence de ma démarche de sorte que les gens ne pensent pas que je suis un tire-au-flanc ou quelque chose du genre» (jeune homme de la rue, 21 ans). Les participants à l'étude ressentent le fait que même la pratique du squeegee permet un sentiment d'appartenance à la société et lui apporte une contribution. Plus spécifiquement, un jeune raconte à quel point son activité informelle l'a convaincu de son statut de citoyen utile:

Des trucs comme la pratique du squeegee m'aident juste à me sentir plus comme, je ne sais pas, plus comme un membre de la société, je suppose, même si ce n'est pas vraiment comme une job formelle. Je ne paie pas de taxes mais je me sens encore comme quelqu'un qui fait quelque chose, tu sais. (Jeune homme de la rue, 19 ans) 
En outre, certains jeunes racontent comment, une fois qu'ils ont gagné de l'argent, ils se sentent davantage appartenir à la société et par conséquent ils se donnent le droit de dépenser de l'argent qu'ils ont gagné au même titre que n'importe qui d'autre. Comme un jeune l'explique: «Je ne me sens pas comme [si je devais me sentir mal] vu que j'ai travaillé pour ça, c'est mon argent après tout et personne ne peut me dire quoi faire avec mon argent, j'ai travaillé pour ça» (jeune homme de la rue, 17 ans).

La plupart des jeunes interrogés travaillent en vue de créer un futur pour eux-mêmes. Quand il leur a été demandé ce que signifiait pour eux «avoir une belle vie», la plupart des jeunes revenaient sur des valeurs traditionnelles comme la stabilité, le soutien social et la sécurité; bref, des valeurs identiques à celles des autres citoyens dans la société en général:

De la nourriture dans mon frigo. Une place où me réveiller qui m'appartienne, avec de la nourriture dans mon frigo et aucun souci... Avoir mes besoins pris en charge au moins minimalement - qu'à mon réveil, je n'aie pas à faire les tâches emmerdantes qui pour l'instant sont nécessaires pour que je puisse satisfaire mes besoins les plus élémentaires. (Jeune femme de la rue, 22 ans)

Certains des participants disent vouloir avoir une «bonne job» qui les aiderait à se bâtir une vie d'adulte (peut-être avec conjoint-e et enfants) et à acquérir des biens classiques, tels qu'une maison et une voiture: «L'argent, tu sais, compte en banque, belle maison, voitures, tu sais. Une femme sur les bras, tu sais, deux, trois enfants, un petit chien... tu sais, un style de vie normal quoi» (jeune homme de la rue, 19 ans). D'autres parlent moins d'une vie conventionnelle et plus d'une manière de vivre spirituelle et proche de la nature, une manière de vivre qui s'ancrerait dans des philosophies résolument alternatives aux valeurs partagées aujourd'hui par le plus grand nombre (par exemple, un mode vie beaucoup plus communautaire):

Mon idéal, ce serait de travailler juste ce qu'il faut pour me payer une parcelle de terre et me procurer le matériel nécessaire pour construire une ferme ou quelque chose dans le genre. Démarrer petit avec juste un peu de bétail, ensuite faire peu à peu fructifier l'affaire, cultiver du cannabis, juste tenir de cette façon et alors là, avoir une famille. (Jeune femme de la rue, 18 ans) 
En dialoguant avec les gens et en partageant avec eux leurs histoires de vie personnelles, les jeunes de la rue ont par ailleurs eu l'occasion de réfléchir à leurs expériences et, dans certains cas, ont même ressenti de la fierté pour avoir réussi à survivre dans des conditions précaires:

$\mathrm{Si}$ tu y vas pour soutirer un billet à quelqu'un, tu vas toujours lui raconter ton histoire et tu vas alors chercher non pas à la réécrire mais bon, juste un peu nettoyer, tu sais, certains aspects du récit de ton voyage. En fait, ce que tu racontes à la personne, c'est un peu la partie dont tu es fier, tes accomplissements quoi. Alors que j'étais sans le sou, j'ai vécu bien plus d'aventures à travers le pays que bien des gens qui avaient pourtant de l'argent pour les vivre, ces aventures. Tu sais, je n'avais peut-être que mes pieds pour voyager mais j'en ai vu bien plus que beaucoup d'autres. (Jeune homme de la rue, 21 ans)

Comment le travail informel se combine avec les efforts de la société pour rendre le pauvre «invisible»?

Vivre sans domicile fixe tout en travaillant informellement peut conduire les jeunes de la rue à exprimer leur créativité (par exemple, faire et vendre de l'art, chanter ou jouer d'un instrument dans la rue), mais pour la plupart d'entre eux, s'engager dans diverses formes de travail informel, c'est d'abord une façon de montrer à la société qu'ils sont non seulement des citoyens mais aussi qu'ils sont disposés à assumer leurs responsabilités pour s'en sortir par eux-mêmes: «Si je ne fais rien, je me sens juste comme une merde, c'est comme si je ne pouvais plus me supporter moimême, je tombe vite dans la déprime quand je ne fais rien un long moment, tu te sens juste inutile» (jeune homme, 19 ans).

Pour beaucoup de ces jeunes participants à l'étude, être capable de travailler informellement est une stratégie pour combattre la négativité qu'ils rencontrent chaque jour. L'idée d'un travail informel comme «activité faite en vue de» a aidé certains jeunes à se construire une bonne estime de soi. Le travail particulier qu'ils ont choisi pouvait en outre cadrer avec la façon dont ils avaient compris leurs rôles et leurs identités en tant qu'individus sans-abri et pauvres. Plus précisément, ces jeunes gens qui sont identifiés comme squeegee considèrent leur travail comme un service auquel le public peut choisir de recourir et voient ce genre d'activité (à savoir, fournir un service ou des biens en échange d'une somme d'argent) comme bien plus honorable que la «simple mendicité». Certains des jeunes qui réfléchissent à leur travail informel 
le décrivent même comme un «divertissement payant». Au même titre que la pratique du squeegee, le rôle de «saltimbanque» comporte aussi un échange de services ou de biens contre de l'argent (par exemple, la chanson d'un chanteur, le poème d'un poète, l'œuvre d'art d'un artiste, la blague d'un humoriste, etc.).

Alors que plusieurs des jeunes interrogés mentionnent des rencontres positives avec les passants, le stigmate social et la discrimination qu'ils subissent quotidiennement à cause de leur instabilité résidentielle et de leur pauvreté érodent souvent leur sentiment d'être des citoyens. La plupart de ces jeunes rapportent que si le travail informel constitue leur dernière option pour se faire de l'argent, pour beaucoup, c'est en fait la seule option quand on a faim, qu'on vit dans l'extrême pauvreté et en marge de la société:

J'ai atterri dans cette grande ville. Je ne pouvais pas y trouver de job... Du coup j'ai dû trouver d'autres façons de me faire de l'argent. Je voulais travailler, mais ce n'était juste pas possible... La mendicité est alors vite devenue ma dernière option. (Jeune homme de la rue, 21 ans)

La réalité pour de nombreux jeunes interrogés, c'est qu'en fin de compte, seul ce type d'activité est accessible pour celles et ceux qui combinent jeunesse, pauvreté, faible éducation, sous-alimentation et vêtements en lambeaux. Une telle situation conduit malheureusement à l'exclusion de ces jeunes de la scène publique, tant sur le plan social que politique et économique.

Le travail informel a rempli plusieurs fonctions pour ces jeunes: 1) Il leur a permis de garder le contrôle sur leurs journées et a assuré qu'ils aient la liberté de travailler un peu plus ou un peu moins selon leurs préférences. 2) Il a fourni un fonds immédiat pour les nécessités (par exemple, nourriture, médicaments, refuge): «J'irai m'asseoir au coin de la rue comme squeegee, ou alors je chanterai, je mendierai ou n'importe quoi du genre et ramasserai l'argent dont j'ai besoin» (jeune femme de la rue, 17 ans). 3) Dans certains cas, le travail informel leur a permis même d'épargner en vue de s'offrir des produits de survie spécifiques (par exemple, de nouvelles chaussures, un sac à dos, etc.): «J'ai épargné pour mon sac à dos et mon sac de couchage...» (jeune homme de la rue, 20 ans). 4) Il a fourni de l'aide à la famille, aux amis ou aux enfants: "J'ai épargné pour mon chien, j'épargne pour rembourser sa facture de vétérinaire» (jeune femme de la rue, 16 ans). 5) Enfin, le travail informel peut fournir une manière de gagner de l'argent suscep- 
tible d'aider les jeunes de la rue à survivre sans pour autant s'engager dans des activités criminelles. Cela a été souligné par plusieurs participants à l'étude comme quelque chose dont ils étaient fiers:

La plupart des gars que je connais ne sont pas de mauvaises personnes, ils ne volent pas les gens, tu sais. Ils ne sont pas les gens qu'on croit qu'ils sont... Ils ne volent pas vraiment, ils ne vendent pas de drogues, ils ne se prostituent pas... Je me souviens de ce petit gars, il avait cette ligne de conduite: «Garde ça clair, c'est mieux de mendier que de voler.» (Jeune homme de la rue, 21 ans)

Le travail qui reste le plus accessible à ces jeunes de la rue renvoie souvent aux activités les plus déplaisantes. À cela s'ajoute l'absence de sécurité d'emploi. Enfin, un tel travail met ces jeunes à la merci de l'opinion publique et d'une surveillance continue. La mendicité et la pratique du squeegee rejoignent d'autres activités ingrates auxquelles les jeunes recourent pour survivre et qui sont considérées comme déviantes, voire criminelles. De telles allusions ne reconnaissent pas les souffrances physiques et sociales qu'endurent ces jeunes dans leurs soucis d'assurer leur survie quotidienne:

Quand tu es exposé, tu prends les vexations et les coups directement en pleine face... beaucoup de gens rendent la vie des squeegees infernale au prétexte «qu'on ne veut pas faire partie de la société», ce qui est faux. Nous faisons partie de la société, nous sommes dehors tous les jours. Qu'est-ce que cela peut bien vouloir dire sinon que nous sommes bel et bien une partie importante de la société? (Jeune homme de la rue, 19 ans)

Dans l'esprit de nombreuses personnes et de l'opinion publique en général, le fait que des jeunes restent ainsi dans la rue est interprété à la fois comme situation d'anomie (au sens d'auto-exclusion sociale) et comme volonté de rupture par rapport à la société, une interprétation qui se traduit alors par des jugements agressifs, une surveillance continue et des harcèlements perpétuels:

Quand tu travailles dans l'espace public, il n'y a plus aucune intimité dans ta vie. On me suivait souvent pour voir où j'allais après avoir tourné le coin, pour voir comment et où je dépensais mon argent. Enfin, tu vois, ce genre de violation de ton intimité, quoi. (Jeune homme de la rue, 18 ans)

Plusieurs des jeunes interrogés pensent que leur volonté de travailler dur devrait être une caractéristique davantage prise en compte par l'opinion publique. Or, elle passe encore trop souvent inaperçue. 
Nombre d'entre eux expliquent ainsi qu'ils ne sont pas tant contrariés par le fait que les gens ne leur donnent pas d'argent (ils voient cela comme un choix personnel et ne tiennent pas rigueur à celles et ceux qui ne leur donnent rien). Ce qu'ils ont surtout du mal à accepter, c'est d'être ignorés, mal compris ou encore accusés d'être des délinquants ou des criminels. Ils répètent souvent qu'ils seraient heureux de voir le public, peu importe qu'il fasse ou non un geste (leur donner de l'argent) à leur égard, essayer de comprendre comment et pourquoi ils ont fini dans la rue. Bref, ils seraient heureux de voir les passants engager davantage la conversation avec eux et être finalement une partie de la solution à leurs problèmes, plutôt que simplement un porte-parole supplémentaire pour l'ignorance, voire la criminalisation de leur existence «publique»:

Depuis que la loi a été adoptée, je suppose que la communauté estime que c'est à elle de prendre la job en main. Tu as de plus en plus de gens qui sont de plus en plus agressifs, qui bondissent hors de leur voiture avec leur clé anglaise et qui essaient de te battre ou des trucs du genre. (Jeune homme de la rue, 20 ans)

Les participants à l'étude ont longuement évoqué le fait d'être l'objet d'une surveillance publique continue et de harcèlements perpétuels. Ils ont décrit les effets de la récente loi sur les «rues plus sûres» qui entrave leurs possibilités de s'engager dans des économies informelles. Le projet de loi 7, qui a pris le statut de loi durant la collecte des données, a amendé la Loi sur les vébicules à moteur en interdisant à quiconque de s'éloigner du trottoir pour «arrêter ou approcher un véhicule moteur dans le but d'offrir, vendre ou fournir des biens ou services au conducteur ou à n'importe qui dans le véhicule» (Projet de loi 7, Loi sur les véhicules à moteur, Nouvelle-Écosse, 2007). Les participants à l'étude ont également décrit les stratégies créatives qu'ils ont utilisées pour éviter de recevoir des amendes, en dépit du caractère très public de leur travail. Enfin, ils soulignent que dans les conditions de survie dans lesquelles ils vivent, ils continuent à vivre de leur travail informel même si cela signifie une augmentation accrue du risque de se faire arrêter:

[...] la plupart des jeunes maintenant, ils disent six up. Comme ça, ils ont toujours des yeux derrière le dos pour voir la police et ils se couvrent les uns les autres. Je pense que c'est la chose la plus importante quand tu es exclu, parce que si [le public] peut te voir, les policiers peuvent te voir aussi. (Jeune homme de la rue, 20 ans) 
Comme le squeegee, pratique très visible, est devenu illégal, les jeunes de la rue trouvent d'autres manières, moins visibles et moins risquées, de combler leurs besoins. En imposant des amendes pour le squeegee, on a en fait poussé certains jeunes vers les aspects les plus durs de l'économie de rue, tels que le trafic de drogue, le crime organisé, le travail du sexe et le vol. Dans la plupart des cas, ce n'est pas du tout ce dans quoi les jeunes souhaitaient tomber: «Ils essaient de nous retirer la pratique du squeegee comme manière de gagner notre vie. Or, si nous n'avons plus cela, qu'allons-nous devenir? Voler des choses comme des voitures ou des maisons? Non. Nous ne voulons pas faire cela. Au moins, comme squeegees, nous ne volions rien ni personne» (jeune homme, 19 ans).

\section{Conclusions}

Plusieurs constats peuvent être dressés quand nous considérons les expériences que ces jeunes vivent par rapport à divers scénarios où il est question de travail mais aussi de «petits boulots». D'abord, les histoires des jeunes de notre étude soulignent la criminalisation de certains types de pauvreté - dans ce cas-ci, une grande partie du travail informel dans lequel les jeunes sans-abri s'engagent. Plutôt que de reconnaître la ténacité de jeunes qui se mettent eux-mêmes en situation vulnérable, souvent à la suite de violences et autres traumatismes familiaux mais aussi sans soutien pour leur permettre une transition saine vers l'âge adulte, la société investit son énergie et une part de nos revenus dans une gestion pénale de la pauvreté des jeunes. La législation qu'on retrouve dans les diverses lois promettant des «rues sûres» et qui a émergé dans de nombreuses villes d'Amérique du Nord, impose des amendes aux jeunes et ils sont pourchassés et arrêtés fréquemment. Une telle législation, à laquelle échappent habituellement des organismes plus conventionnels comme les œuvres de charité ${ }^{3}$, paraît peu préoccupée par toutes les difficultés liées au recours à une activité informelle. Elle semble bien davantage soucieuse de rendre invisibles certaines populations aux yeux des populations intégrées (Hunter, et al., 2009) même si paradoxalement, la société en général applaudit souvent la

3. Par exemple la section 13 du projet de loi 7 concernant la loi modifiée sur les véhicules à moteur, Nouvelle-Écosse, 2007 (loi déjà présentée plus haut) laisse clairement entendre que le changement «ne s'applique pas aux activités de collecte de fonds». 
résilience affichée par les communautés qui s'engagent dans le travail informel (pensons aux systèmes de troc) quand l'économie souffre (par exemple, en période de récession et de chômage sévère) (Reimer, 2000).

La seconde série de difficultés affrontées par les jeunes révélées dans cette étude a trait aux énormes défis associés au maintien d'un travail formel alors qu'on est sans-abri. Ces pressions collectives, incluant les obstacles à un emploi formel et une législation qui définit comme illégale la plupart sinon tous les revenus générés par les activités de ces jeunes gens (quand ça ne l'est pas dans la lettre du droit, ça l'est au moins dans l'esprit), ont été vues comme poussant les jeunes de la rue vers une mendicité passive et par défaut (O'Grady et Greene, 2003). Quant à l'augmentation des crimes violents et des crimes contre la propriété, ils ont été interprétés comme la conséquence non seulement de l'iniquité des marchés du travail mais aussi de la difficulté à obtenir les fonds d'aide adéquats (Baron et Hartnagel, 2002).

Par ailleurs, les histoires racontées par les jeunes de la rue suggèrent que les espaces publics appartiennent seulement au monde de l'entreprise, plutôt qu'au «public, tout le public, et ce malgré les protections des droits de la personne tels que définis dans la Déclaration universelle des droits de l'bomme et dans la Charte canadienne des droits et libertés" (Hunter et al., 2009: 8). C'est ainsi que le monde de l'entreprise a le pouvoir de neutraliser les droits des plus vulnérables de notre société (à savoir, celles et ceux qui sont jeunes, pauvres et qui fuient un traumatisme familial). Simplement parce que les jeunes de la rue rendent mal à l'aise la plupart d'entre nous?

Ces observations soulèvent plusieurs questions fondamentales. Comment davantage conscientiser la société civile tant sur les causes profondes de la pauvreté que sur les capacités et autres aptitudes des jeunes itinérants? Comment faire prendre conscience à l'opinion publique que ces jeunes n'ont pas peur de travailler et qu'ils regrettent juste qu'on leur rende cette tâche si difficile? Comment encourager les communautés et les écoles à soutenir les jeunes «à risque» de façon à les garder à l'école alors qu'ils connaissent une instabilité résidentielle? Comment pourraient-elles aider ces jeunes à trouver un logement convenable? Enfin, comment inviter l'opinion publique à reconsidérer de façon critique qui sont les véritables destinataires de lois instituées au nom de rues plus sûres, en particulier à la lumière des expériences de jeunes gens telles qu'elles ont été relatées dans cette étude? 
Des réponses à de multiples niveaux sont nécessaires si on veut comprendre les raisons sous-jacentes pour lesquelles les jeunes de la rue travaillent informellement. Imposer des amendes à ces jeunes ne suffit pas à les en dissuader. On ne touche pas là en effet aux circonstances de l'appauvrissement et de l'instabilité résidentielle qui créent ultimement les situations dans lesquelles le travail informel devient la seule option possible. Les décideurs politiques et les agents du maintien de l'ordre doivent dès lors interroger la futilité d'une telle approche. Ils ne doivent plus criminaliser les tentatives de survie de ces jeunes mais plutôt développer des outils susceptibles de combattre les principales causes de la pauvreté (notamment, en améliorant les déterminants sociaux de la santé). Nous avons montré que le maintien d'un emploi pouvant combiner travail (formel) et instabilité résidentielle passe par une remise en question des perceptions publiques, qu'elle exige une conscientisation et une éducation de l'opinion publique à propos des comment et des pourquoi de la pauvreté, qu'elle doit encourager le public et les jeunes de la rue à trouver, ensemble, des solutions. Quand on demande à ces jeunes ce qui pourrait être fait pour améliorer leur situation, nombre d'entre eux suggèrent que le public a d'abord besoin d'être informé. Ils voient là une solution possible à la diminution du harcèlement dont ils sont régulièrement victimes mais aussi un frein à l'ignorance qu'ils affrontent tous les jours dans leurs interactions avec le public. Il apparaît en fait que même les dirigeants politiques - pourtant souvent considérés plus informés que le «public en général»- comprennent mal la complexité de la problématique des jeunes de la rue. La promotion d'emplois qui bénéficieraient du soutien des dirigeants politiques, des chefs d'entreprise et de la communauté au sens large pourrait créer de nouvelles opportunités pour les jeunes.

Enfin, nous estimons essentiel que tant les membres de la communauté que les intervenants (décideurs politiques, fournisseurs de services et organismes communautaires) s'engagent dans la formulation des réponses aux enjeux de la pauvreté et de l'instabilité résidentielle. Alors que ces phénomènes (la pauvreté, l'instabilité résidentielle) sont souvent considérés comme faisant partie d'une problématique qui ne concerne qu'une minorité d'individus, ils affectent en fait tout le monde. Des montants significatifs prélevés sur l'argent des contribuables sont en effet utilisés chaque année pour gérer le problème des jeunes de la rue. Une grande partie de ces fonds est distribuée dans les secteurs gouvernementaux tels que les services sociaux, la justice, l'éducation et la santé. 
Si ces programmes ont besoin de ces fonds et les méritent, l'objectif doit toutefois rester la garantie d'un retour sur investissement pour les citoyens mobilisés à cette fin. Or, plus une grande partie de la communauté s'intéressera aux enjeux de pauvreté et d'instabilité résidentielle, plus on multipliera les chances de voir les décideurs politiques s'engager à leur tour à trouver des solutions plus adéquates et plus efficaces non seulement pour aider les jeunes en situation de vulnérabilité (dans la rue, sans-abri) mais aussi pour leur permettre de se réaliser.

Alors que les expériences et les intuitions des jeunes qui vivent dans la rue comme de ceux qui les soutiennent et en prennent soin décrivent la complexité et les nuances entourant les imbrications entre manque de logement et de travail, le grand récit des opinions dominantes semble bien moins compliqué et intriqué. Les mythes populaires qu'on retrouve chez le grand public suggèrent en effet que la jeunesse de rue est dépourvue d'intelligence, paresseuse et délinquante. Or, ces jeunes apparaissent réfléchis, ils disposent d'une éthique de travail forte qui est ancrée dans la société civile, et non en dehors d'elle. Loin d'être des déviants ou des criminels par nature, les jeunes de la rue qui ont participé à l'étude sont davantage enclins à chercher, dans le contexte qui est le leur, un emploi légal et légitime. Et c'est là, semble-t-il, les conclusions centrales de notre article: si ces jeunes ne trouvent pas facilement de travail formel, c'est parce qu'il n'y a pas suffisamment d'incitations économiques qui leur sont offerts. Ils n'ont dès lors d'autre choix que de se tourner vers des activités informelles qui, d'une part, leur fournissent de l'argent pour survivre et, d'autre part, leur apportent une certaine fierté, une estime de soi et un sentiment d'accomplissement et ce, en dépit de la dévalorisation, du harcèlement et des moqueries qui accompagnent de telles activités. Pour les jeunes, le travail informel renvoie à une décision rationnelle de survie. Pour certains d'entre eux, il correspond à un style de vie fait de voyages et d'indépendance; pour d'autres, il offre un soutien quotidien pour survivre dans une culture de la rue. Ce qui est troublant en ce qui concerne le travail informel, c'est que s'il continue à condamner les jeunes de la rue à un statut de marginal et qu'en ce sens, il continue à les exclure socialement, il constitue aussi, pour eux, un moyen de survie. 


\section{Références}

Alleva, F. (1988). Youth at risk, systems in crisis: a dialogue with youth who needed shelter. [Dissertation]. Boston: Boston University.

Auerswald, C, \& Eyre, S. (2002). Youth homelessness in San Francisco: A life cycle approach. Social Science or Medicine, 54, 1497-1512.

Baron, S. W. (2001). Street youth labor market experiences and crime. Canadian Review of Sociology/Revue canadienne de sociologie, 38 (2), 189-215.

Baron, S. W. (2004). Street youth, strain theory and crime. Journal of Criminal Justice, 34 (2), 209-233.

Baron, S. W., \& Hartnagel, T. F. (2002). Street youth and labor market strain. Journal of Criminal Justice, 30, 519-533.

Bill No. 7 (2007). 2nd Session, 60th General Assembly, Nova Scotia, 56 Elizabeth II, 2007, Motor Vehicle Act (amended), CHAPTER 45 OF THE ACTS OF 2007, An Act to Amend Chapter 293 of the Revised Statutes, 1989, the Motor Vehicle Act, Royal Assent: December 14, 2007, http://www.gov. ns.ca/legislature/legc/bills/60th_2nd/3rd_read/b007.htm

Coates, J. (2000). No Place at the Table: A Study of Homelessness in Fredericton. Fredericton NB: Department of Family and Community Services.

Crow, D., \& Albo, G. (2005). Neo-liberalism, NAFTA, and the state of the NorthAmerican labor movements. Just Labour, 6/7,12-22.

Dachner, N., \& Tarasuk, V. (2002). Homeless "squeegee kids": Food insecurity and daily survival. Social Science $\mathcal{O}$ Medicine, 54 (7), 1039-1049.

Durham, K. (2003). Housing Youth : Key Issues in Supportive Housing. Corporation for Supportive Housing, http://documents.csh.org/documents/pd/youth/ HousingYouthKeyIssues.pdf.

Faux, J., Salas, C., Campbell B., \& Scott, R. E. (2006). Revisiting NAFTA : Still not working for North America's workers. EPI Briefing Paper, 173. Washington, DC: Economic Policy Institute.

Gaetz, S. (2004). Safe streets for whom? Homeless youth, social exclusion, and criminal victimization. Canadian Journal of Criminology and Criminal Justice, 46 (4), 423-456.

Gaetz, S., \& O'Grady, B. (2002). Making money: Exploring the economy of young homeless workers. Work, Employment and Society, 16 (3), 433-456.

Hagan, J., \& McCarthy, B. (1997). Mean Streets: Youth Crime and Homelessness. Cambridge: Cambridge University Press.

Halifax Regional Municipality (2004). Homelessness in HRM : A Portrait of Streets and Shelters. Halifax, NS: Planning and Development Services, Halifax Regional Municipality.

Hughes, J. Broom, B., Cakmak, S., Clark, S., Keeler, C., LeMoine, D., MacInness, M., Pratt, A., Wood, B. (2005). Youth Matters: A Mental Health Needs Assessment of Homeless Youth. Canadian Conference on Homelessness. York University, Toronto, mai 2005.

Hunter, G., Belton, A., Johnson, T., \& Pederson, S. (2009). Regina's Ban on Panhandling: Sweeping the Poor and Homeless off the Streets. Social Policy 
Research Unit, Poverty Paper 3. Regina : Faculty of Social Work, University of Regina.

Karabanow, J. (2004a). Being Young and Homeless: Understanding How Youth Enter and Exit Street Life. New York: Peter Lang Publishing INC.

Karabanow, J. (2004b). Exploring Salient Issues of Youth Homelessness in Halifax, Nova Scotia. Ottawa, ON: Report available through Human Resources Development Canada Supportive Communities Partnership Initiative.

Karabanow, J., Clement, P., Carson, A., \& Crane, K. (2005). Getting off the street: Exploring strategies used by Canadian youth to exit street life. Ottawa, ON: The National Homelessness Initiative, National Research Program.

Karabanow, J. (2006). Becoming a street youth: Uncovering the stages to street life. Journal of Human Behavior in the Social Environment, 13 (2), 49-72.

Karabanow, J., Hopkins, S., Kisely, S., Parker, J., Hughes, J., Gahagan, J., \& Campbell, L. A. (2007). Can you be healthy on the street? Exploring the health experiences of Halifax street youth. Canadian Journal of Urban Research, $16(1), 12-32$.

Karabanow, J. (2008). Getting off the street: Exploring young people's street exits. American Behavioral Scientist, 51 (6), 772-788.

Karabanow, J. Ticknor, J., Hughes, J., Kidd, S., \& Patterson, D. (2009). Working within Formal and Informal Economies: How Homeless Youth survive in Neo-liberal Times. HRSDC report.

Krauss, D., Eberle, M., \& Serge, L. (2001). Environmental Scan on Youth Homelessness: Final Report. Ottawa, ON: Canada Mortgage and Housing Corporation.

Losby, J. L., Else, J. F., Kingslow, M. E., Edgcomb, E. L., Malm, E. T., \& Kao, V. (2002). Informal Economy Literature Review. Vancouver, BC: Institute for Social and Economic Development and the Aspen Institute.

McCarthy, J., \& Nelson, G. (1991). An evaluation of supportive housing for current and former psychiatric patients. Hosp Community Psychiatry, 42 (12), 1254-1256.

Novac, S., Serge, L., Eberle, M., \& Brown, J. (2002). On Her Own: Young Women and Homelessness in Canada. Ottawa, ON: Canadian Housing and Renewal Association.

O'Grady, B., \& Gaetz, S. (2004). Homelessness, gender and subsistence: The case of Toronto street youth. Journal of Youth Studies, 7 (4), 397-416.

O'Grady, B., \& Greene, C. (2003). A social and economic impact study of the Ontario Safe Streets Act on Toronto squeegee workers. Online Journal of Justice Studies, 1.

Reimer, B. (2000). The informal economy in rural Canada. Presentation to the Canadian Economy Research Forum.

Schneider, F. (2002). Size and measurement of the informal economy in 110 countries Around the world. Workshop of Australian National Tax Centre. ANU, Canberra, 17 juillet 2002.

Scott, R. E. (2008). Pennsylvania Stagnation: Is NAFTA the Culprit? New York Times, April 15, 2008. Consulté le 12 mars 2009 sur: http://www.epi. org/publications/entry/webfeatures_viewpoints_pa_jobs_and_nafta/ 
Strauss, A., \& Corbin, J. (1990). Basics of Qualitative Research. Newbury Park: Sage Publications.

Toronto Mayor's Homeless Action Task Force (1990). Taking Responsibility for Homelessness: An Action Plan for Toronto. Toronto: Metro Community Services.

Vogel, D. R. (2006). Harder times: Undocumented workers and the US informal Economy. Monthly Review, 58 (3), 29-39.

ABSTRACT - This paper explores how employment and labor are situated within the lives of homeless youth and is based upon the findings emerging from in-depth interviews with 34 youth in Halifax, Canada. The findings suggest that street-involved and homeless young people are straddling formal and informal work economies while mediating layers of external and internal motivations and tensions. For the most part, youth interviewed engaged in informal money-making activities including panhandling, squeegeeing, busking, making and selling art, performing poetry and/or jokes, among many other creative pursuits. The diversity of these activities demonstrates the entrepreneurial spirit and creativity/resilience of street-involved young people. They are willing to undertake any number of often undesirable tasks to survive. The reality is that the participants in this study could not very easily engage in formal work. In addition, their stories showed how society takes particular effort to criminalize certain types of poverty - in this case much of the informal work in which homeless youth engage.

KEYWORDS - Street youth, employment, survival strategy, deviance, informal economy.

RESUMEN - Con base en 34 entrevistas a fondo con jóvenes de Halifax, Canadá, el presente artículo analiza el lugar que el trabajo formal e informal ocupan en las vidas de los jóvenes de la calle. La investigación sugiere que los jóvenes que habitan o realizan actividades en la calle oscilan entre las economías formal e informal, con diversas mediaciones, motivaciones y tensiones internas y externas. La mayoría de jóvenes entrevistados realiza actividades cuyos ingresos son informales, entre ellas la mendicidad, el limpiado de parabrisas y diversas actividades creativas como la interpretación musical, la fabricación y venta de arte y la presentación de poesía y comedia, entre otras. Esta diversidad de actividades muestra el espíritu empresarial y la creatividad y perseverancia de los jóvenes de la calle, dispuestos para sobrevivir a emprender actividades muchas veces consideradas indeseables. Una realidad es que los participantes de este estudio no podrían fácilmente obtener empleo formal. Además, lo que cuentan ilustra la forma en que la sociedad se empeña en criminalizar cierto tipo de pobreza, en este caso el trabajo informal en que los jóvenes de la calle se autoemplean.

PALABRAS CLAVE • Jóvenes de la calle, empleo, estrategia de supervivencia, desviación, marginalidad, economía informal. 